\title{
Computersimulation von Wendelförderern mit Hilfe der Diskrete Elemente Methode (DEM)
}

\author{
DR.-ING. A. MINKIN \\ INSTITUT FÜR FÖRDER- UND BAUMASCHINENTECHNIK, STAHLBAU, LOGISTIK (IFSL), LEHRSTUHL FÜR \\ FÖRDERTECHNIK, OTTO - VON - GUERICKE -UNIVERSITÄT MAGDEBURG
}

\section{Zusammenfassung}

Wendelfördersysteme werden vorrangig in Kläranlagen für Rechengut und Klärschlamm, in der Abfallverwertung und -entsorgung, in der Holzindustrie (Späne und Hackschnitzel), in der Lebensmittelindustrie und Landwirtschaft (Saatgut), in der Zellstoff-, Papier- und Bauindustrie (Mörtel) u. a. eingesetzt. In der modernen Kreislaufwirtschaft und in der Altlastensanierung (Altdeponieaufbereitung u. a.) ist der Einsatz von Wendelförderern besonders angezeigt. Somit ist die Bedeutung des Wendelförderers als Gliedes in der logistischen Kette der Abfallverwertungsbetriebe sehr groß. Der Vortrag erläutert die Rolle des Wendelförderers in der logistischen Kette der Entsorgungswirtschaft. Es werden die Ergebnisse der experimentellen Untersuchung sowie der DEMSimulation von Wendelförderern vorgestellt und diskutiert, welche Chancen und Möglichkeiten sich für eine verbesserte Auslegung des Wendelförderers ergeben.

\begin{abstract}
Spiral conveyors are mainly used in clarification plants for screenings and sludges, in waste recycling and disposal, in the timber industry (wood splints and chips), in the food industry and agriculture (seeds), in the cellulose, paper and construction industry (plaster), etc.. In modern closed loop recycling management and cleanup operations (processing of dumpsites, etc.) spiral conveyors are of special importance and an important link within the logistic chain of a waste recycling plant. The lecture describes this role and presents the results of experiments. Furthermore, it deals with the DEM simulation of spiral conveyors and points out the chances and possibilities opened up by an improved design of spiral conveyors.
\end{abstract}

\section{Einleitung}

Das Kreislaufwirtschaft- und Abfallgesetz (KrW-/AbfG) hat auch für fördertechnische Aufgabenstellungen zahlreiche Auswirkungen. Diese Entwicklung wird im Zuge der Durchsetzung weiterer Rücknahmeverordnungen anhalten und insbesondere zu steigenden Anforderungen im Bereich der Abfall- und Entsorgungslogistik führen. Im Bereich der Materialflußtechnik sind damit zahlreiche neue Förderprinzipe und Auslegungsmethoden zu entwickeln, die für meist sehr differenzierte, nicht exakt zu definierende und zudem sehr inhomogen Fördergüter eingesetzt werden müssen. Zusätzlich ist davon auszugehen, daß von einigen Fördergütern schädliche Umweltauswirkungen ausgehen, deren Emissionen beim Förderprozeß in der Logistikkette der Abfallwirtschaft verhindert werden müssen.

Die Wendelförderer haben sich für konventionelle Förderaufgaben mit speziellen Anforderungsprofilen bisher praktisch bewährt. In den letzten Jahren nimmt die Bedeutung des Wendelförderers als Gliedes in der logistischen Kette der Abfallverwertungsbetriebe immer stärker zu. Obwohl die theoretischen und experimentellen Untersuchungen an Wendelförderern bereits durchgeführt wurden, könnten die Ergebnisse nur für begrenzte Schüttgutgruppen und Förderergeometrien erzielt werden.

Die Diskrete Elemente Methode, kurz DEM genannt, erlaubt die Berechnung und Simulation von diskreten, diskontinuierlichen Vorgängen im Wendelförderer. Dieses relative neue Simulationsverfahren erlaubt neben einer qualitativen Analyse der Bewegungsvorgänge des Schüttguts auch die quantitative Analyse von Massenströmen, Drehmomenten, Axialkräften an der Wendel sowie der Antriebsleistungen eines Wendelförderers. 


\section{Stand der Forschung}

In den Arbeiten [Krause05a]-[Krause05e], [Minkin05] wurden die neusten Ergebnisse der qualitativen und quantitativen Untersuchungen zur Wendelförderung veröffentlicht.

Für die Forschung zur Wendelförderung wurden zwei aufwendige Versuchsstände, der WendelfördererGroßversuchsstand (GVS) (Abb. 1) und der Wendelförderer-Modellversuchsstand (MVS) (Abb. 2) projektiert und errichtet. Diese stellen die Grundlage für die genannten Arbeiten dar.

Auf Basis der erzielten Forschungsergebnisse konnten der Fördervorgang im Wendelförderer genau beschrieben sowie die Auslegungsrichtlinien für horizontale und leicht geneigte Wendelförderer (HlgWF) in Abhängigkeit vom Schüttguteigenschaften und von den Betriebsparametern formuliert werden.

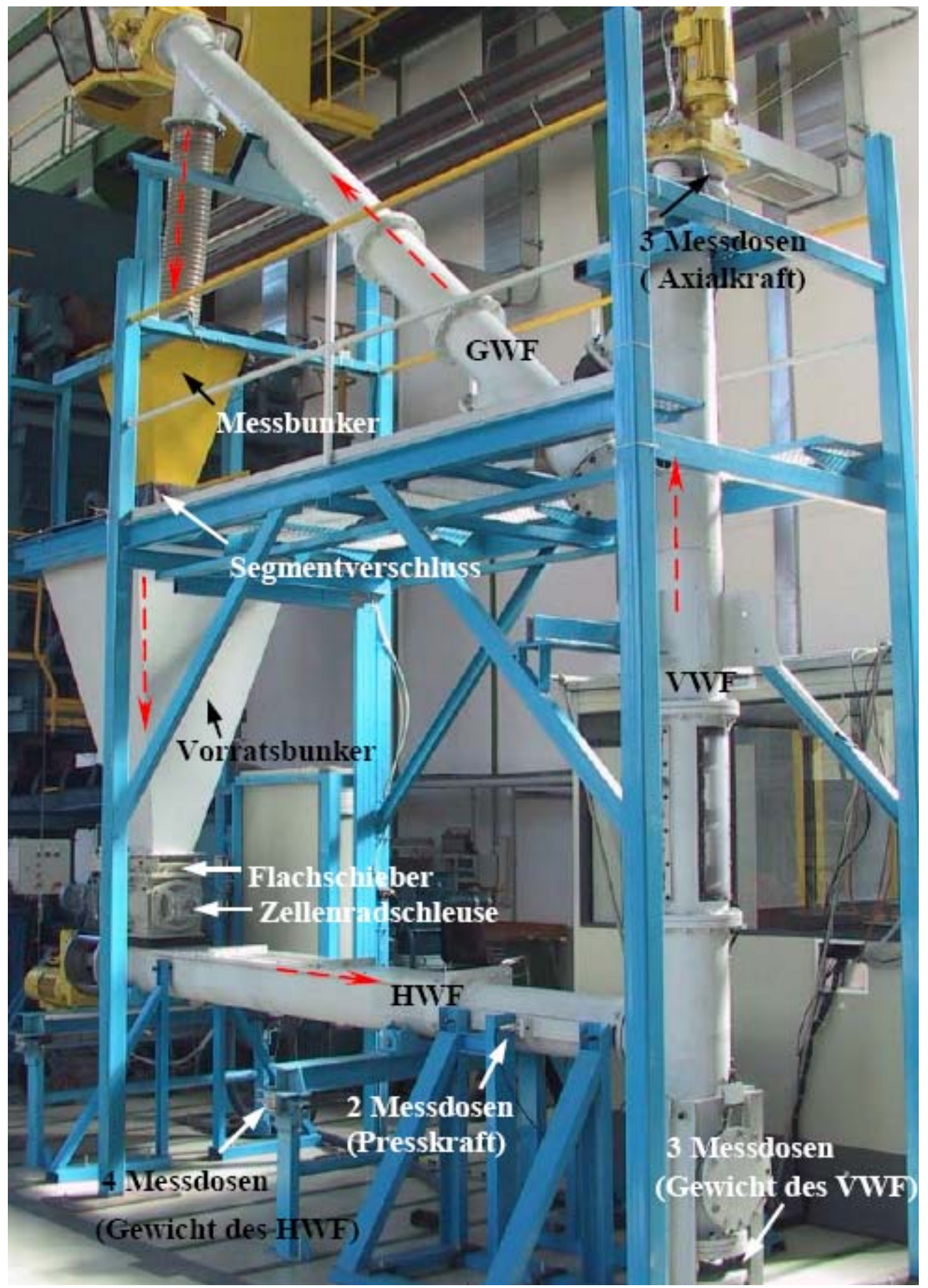

Abbildung 1: Der Wendelförderer-Großversuchsstand (GVS) 


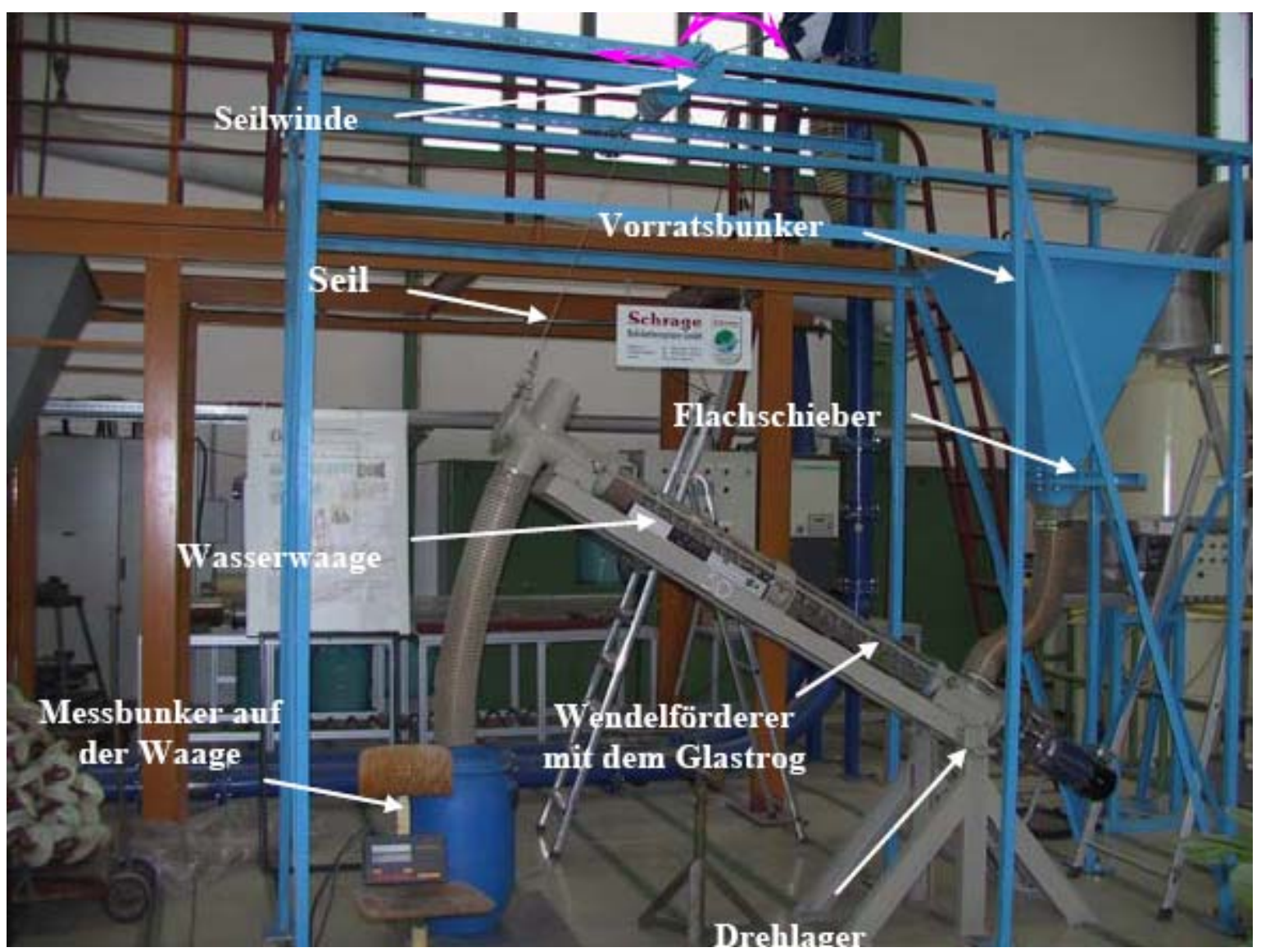

Abbildung 2: Der Wendelförderer-Modellversuchsstand (MVS)

Die Diskreten Elemente Methode (DEM) stellt einen völlig neuen Ansatz dar, um schüttgutmechanische Vorgänge in Förderern erfassen zu können. Sie wurde von Cundall und Strack in den 1970er Jahren entwickelt. Die DEM basiert auf der Lösung der Newtonschen Bewegungsgleichungen aller Partikel eines ruhenden oder auch fließenden Mehrkörpersystems und stellt damit den Gegensatz zur klassischen kontinuumsmechanischen Betrachtungsweise dar. In der geometrischen Gestaltung der Partikel, den Randbedingungen und in der Modellierung der Kontakte bestehen große Freiheiten. Die aus Volumen- und Oberflächenkräften resultierenden Bewegungsgleichungen werden mittels einer expliziten Integrationsmethode gelöst. Demzufolge wird die Veränderung der Konfiguration in äquidistanten Zeitschritten simuliert [Gröger99].

Besondere Verbreitung fand die DEM bereits in der Felsmechanik und Verfahrenstechnik. Ihr Einsatz in der Materialflußtechnik war aus Rechnerkapazitätsgründen bis jetzt begrenzt [Gröger99], [Gröger03], [Katterfeld03], [Katterfeld04].

Die Forschungsergebnisse zum Wendelförderer aus [Krause05a]-[Krause05e], [Minkin05] bilden eine notwendige Basis zur Verifizierung des DEM-Modells für Wendelförderer.

\section{Vorteile der DEM-Simulation für die Auslegung und Dimensionierung von Wendelförderern}

Das in [Krause05a]-[Krause05e], [Minkin05] beschriebene Untersuchungsverfahren am Wendelförderer gehört zu den klassischen Untersuchungsstrategien. Der Nachteil dieser Strategien besteht darin, daß neue Berechnungsmodelle empirische Faktoren beinhalten, die immer nur mit einem großen versuchstechnischen Aufwand ermittelt werden können. Der Grund dafür ist die enorme Komplexität der schüttgutmechanischen Vorgänge im Wendelförderer, die analytisch nicht erfaßt werden können. Darüber hinaus gelten die empirischen Faktoren nur für begrenzte Geometrie- und Betriebsparameter sowie für repräsentative Schüttgüter. Das alles erschwert den Ausbau des Berechnungsmodells für neue Einsatzfälle. Die wirtschaftliche Auslegung der Wendelförderer ist daher eine komplexe Problematik. 
Aus materialflußtechnischer Sicht kann die DEM-Simulation von Wendelförderern als neuartige Untersuchungsstrategie bezeichnet werden. Der Einsatz der DEM bei der Simulation der Fördervorgänge im Wendelförderer hat mehrere Vorteile. Zu denen vor allem zu zählen:

- Kostengünstiger Funktionsnachweis und die Möglichkeit zur Simulation und Visualisierung der Fördervorgänge (auch mechanischer Kennwerte und Massenströme) im Wendelförderer.

- Überprüfung der Problemstellen im Wendelförderer (Festigkeits- und Verschleißprüfung, Gutauf- und abgabestellen).

- Einbeziehung verfahrenstechnischer Prozesse in den simulierten Fördervorgang (Komprimierung, Mischen und Separation).

- Vorteile beim Marketing und Vertrieb der Wendelförderer-Systeme.

Die in [Krause05a]-[Krause05e], [Minkin05] veröffentlichten Forschungsergebnisse sind aber für die Anpassung des DEM-basierten Programmtools für Wendelförderer an die Realität erforderlich. Mit Hilfe des verifizierten DEM-basierten Programmtools wird die Simulation der Fördervorgänge im Wendelförderer möglich, wodurch sich nicht nur das Funktionsprinzip für eine breite Schüttgutpalette überprüfen läßt, sondern auch die wichtigsten mechanischen Kennwerte in Abhängigkeit von Betriebs-, Geometrie- und Schüttgutparametern ermittelt werden können. Eine kostengünstige Möglichkeit zur fehlerfreien Dimensionierung und Auslegung von Wendelförderern für bestimmte Schüttgutgruppen (keine fasrigen Güter), jedoch nahezu alle Betriebsparameter und Förderergeometrien erscheint mit Hilfe der DEM in greifbarer Nähe.

\section{DEM-Simulationsmodell für Wendelförderer}

Die am IFSL laufenden Projekte „Einsatz der Diskrete Elemente Methode in der Schüttgut-Fördertechnik“ und „ENVIS - Entwicklung und Verifizierung innovativer Simulationssoftware zur Auslegung von Schüttgutförderern unter Berücksichtigung sich verändernder Marktbedingungen“" haben das Ziel, DEM-basierte Programmtools für mehrere Stetigförderer zu entwickeln. Unter anderem wird im Rahmen der Projekte auch ein Simulationsmodell für Wendel- und Schneckenförderer erarbeitet.

Die ersten Versuche, den Fördervorgang im Schneckenförderer zu simulieren, wurden bereits von Shimuzu und Cundall (Itasca Group Inc.) in [Shimizu01] unternommen.

Das Simulationsmodell wird mit der Software PFC-3D (Particle Flow Code 3D) von der Firma Itasca erstellt. Das Hauptproblem liegt dabei in der fehlerfreien Formulierung des Kontaktmodells. Dafür sind nämlich die Ergebnisse der experimentellen Untersuchungen am Wendelförderer notwendig.

Bei der Erstellung des Simulationsmodells müssen verschiedene Vereinfachungen (vor allem in der Geometrie, Partikelform und -anzahl) getroffen werden, um eine geringe Rechenzeit bei der Simulation zu gewährleisten, ohne daß der Bezug zur Realität verloren geht. Eine weitere Aufgabe ist die Präzisierung der Materialeigenschaften im Modell.

Bei der Erarbeitung des Modells für einen Wendelförderer wird ein Schnitt aus der Mitte des Förderers betrachtet, wobei die gesamte Förderlänge auf die Länge von zwei Wendelsteigungen reduziert wird. Am Anfang und am Ende des Schnittes werden periodische Grenzen gesetzt, so daß das transportierte Schüttgut aus dem Schnitt-Ende direkt in den Schnitt-Anfang zurückfließt. Dadurch wiederholt sich der Fördervorgang, ohne neue Partikel zu generieren. Solche Abstraktionen ermöglichen die Reduktion der Partikelanzahl und somit auch die Verkürzung der Rechenzeit.

Basis für die Beschreibung der Schüttguteigenschaften im Simulationsmodell bilden die Ergebnisse der Schüttgutuntersuchungen, die im Schüttgutlabor des IFSL unternommen werden.

Bislang bereitet die Wahl der richtigen Simulationsparameter die größten Schwierigkeiten bei der Durchführung von realitätsnahen DEM-Simulationen. Mit Hilfe geeigneter Strategien zur experimentellen Schüttgutanalyse müssen die Simulationsparameter kalibriert werden.

\section{Analyse und Vergleich der Ergebnisse der DEM-Simulation mit der Realität}

Für die Erstellung des DEM-Modells eines horizontalen Wendelförderers wurden die geometrischen Parameter des Wendelförderers des MVS einbezogen, die in [Krause05c], [Krause05e] und [Minkin05] ausführlich beschrieben sind. Bei der Modellierung der Schüttguteigenschaften wurden die Ergebnisse der Schüttgutuntersuchungen für Mischgranulat verwendet. Dabei wird bei der Präzisierung der Schüttguteigenschaften die Rollreibung für die Partikel im Modell berücksichtigt. Der Böschungswinkel auf Basis der kalibrierten Simulationsparameter des modellierten Schüttgutes ist dann etwa gleich dem Böschungswinkel des realen Mischgranulats. 


\subsection{Ergebnisse der qualitativen Untersuchungen mit Hilfe der DEM für horizontale Wendelförderer mit Mischgranulat}

Abbildung 3 zeigt den Fördervorgang im horizontalen Wendelförderer (HWF) des GVS beim Füllungsgrad $\eta_{F} \approx$ 0,6 und der Wendeldrehzahl $n_{W}=16 \mathrm{~min}^{-1}$ während des Transports des Mischgranulats.

Abbildung 4 zeigt die Ergebnisse der DEM-Simulation für die horizontale Stellung des MVS bei $\eta_{F}=0,6$ und $n_{W}=46 \mathrm{~min}^{-1}$ mit 24500 runden Einzelpartikeln. Beim Vergleich beider Bilder können die ersten qualitativen Aussagen getroffen werden.

Wie aus Abbildung 3 zu entnehmen ist, wird das Schüttgut während der Förderung im Beharrungszustand aus der statischen Ruhelage um den Verdrehwinkel $\varepsilon$ in Drehrichtung der Wendel ausgelenkt. Während der Versuche an beiden Versuchsständen wurde auch beobachtet, daß mit wachsendem Füllungsgrad die rotatorische Komponente der Gutbewegung zunimmt.

Vor dem Simulationsstart wurden die Partikel mit originalen Partikeldurchmessern im DEM-Modell aus der Schicht A $\left(\eta_{F} \leq 0,3\right)$ grün und aus der Schicht $\mathrm{B}\left(0,3<\eta_{F} \leq 0,0\right)$ gelb markiert. Nachdem der Beharrungszustand erreicht wurde, nimmt der Schüttgutkörper in der Simulation die gleiche Form (Abb. 4) ein, die er auch während des realen Fördervorganges im Wendelförderer (Abb. 3) einnimmt. Mit dem zunehmenden Füllungsgrad nahm auch in der DEM-Simulation die rotatorische Komponente der Gutbewegung zu. Dabei vermischten sich die Partikel bei $\eta_{F}=0,6$ aus beiden Schichten (Abb. 4). Der beobachtete Fördervorgang in der DEM-Simulation des HWF spricht dafür, daß das DEM-Modell des HWF gut mit den Ergebnissen der qualitativen Untersuchungen am HWF aus [Krause05a]-[Krause05e], [Minkin05] übereinstimmt.

Weiterhin ermöglicht die DEM-Simulation die Pfade der Partikeln (Abb. 5) direkt aufzuzeichnen. Anhand der Radiotrace-Methode kann später im Rahmen des ENVIS-Forschungsprojektes am neuen Versuchsstand geprüft werden, ob der Charakter der Partikelpfade mit der Realität übereinstimmt.

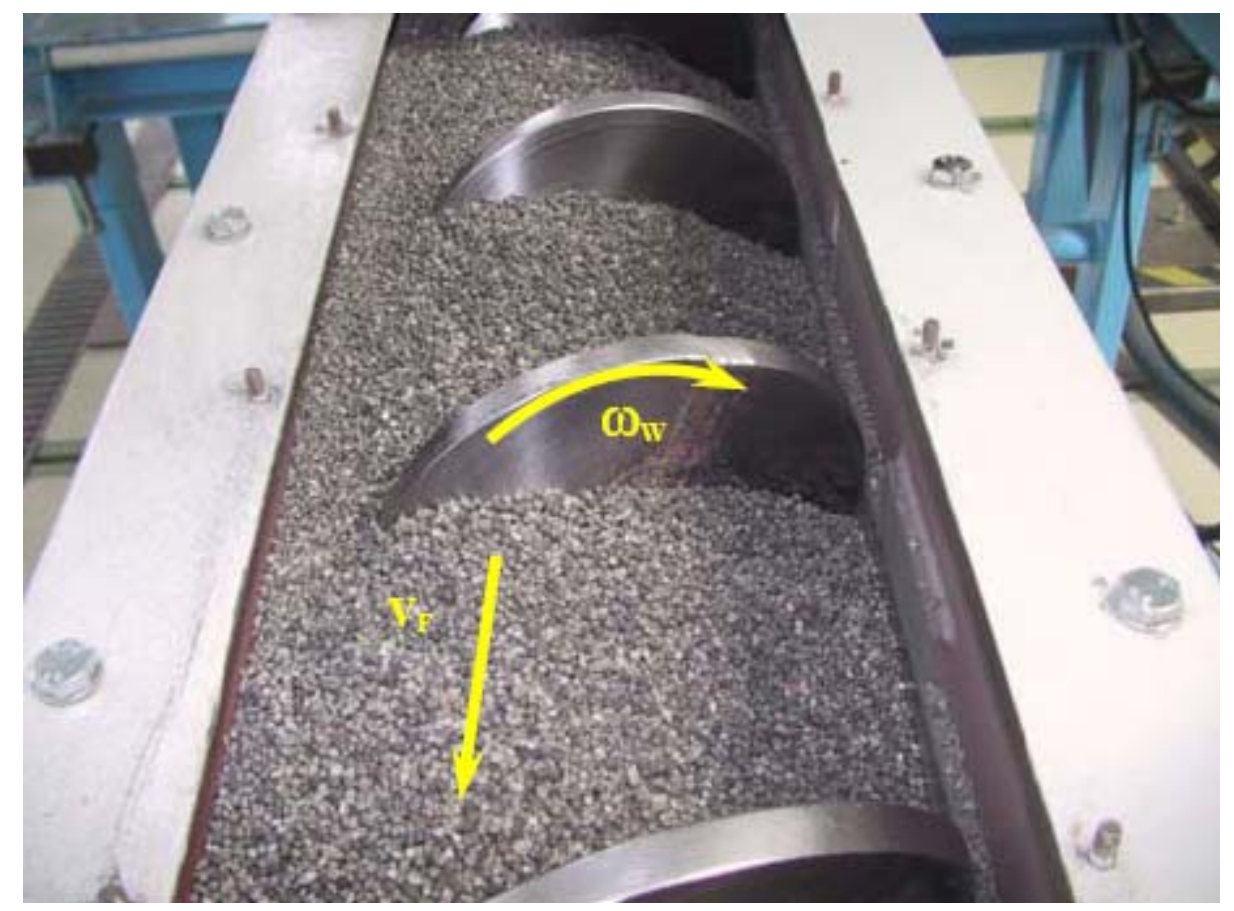

Abbildung 3: Fördervorgang im HWF des GVS bei $\eta_{F} \approx 0,6$ und $n_{W}=16 \mathrm{~min}^{-1} \mathrm{mit}$ dem Mischgranulat 


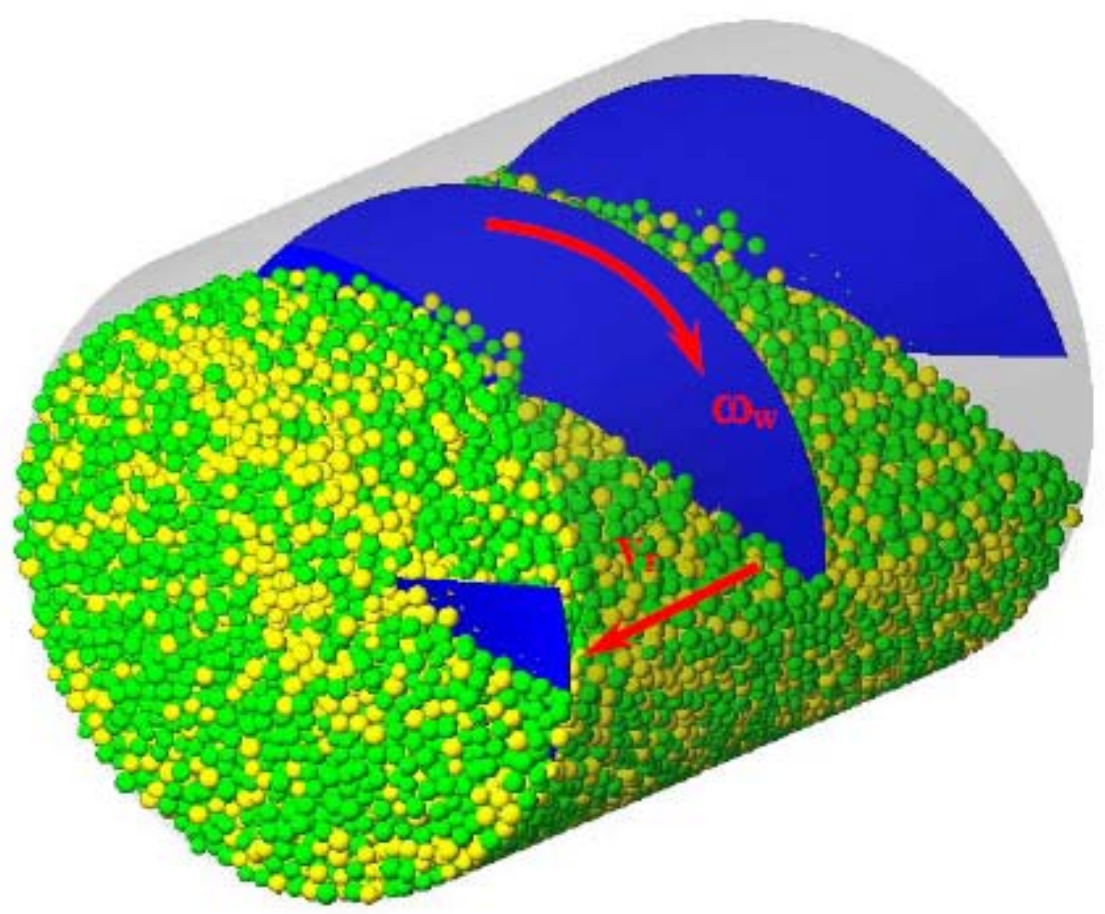

Abbildung 4: DEM-Simulation des horizontalen MVS bei $\eta_{F}=0,6$ und $n_{W}=46 \mathrm{~min}^{-1} \mathrm{mit}$ verschiedenen Partikelgrößen

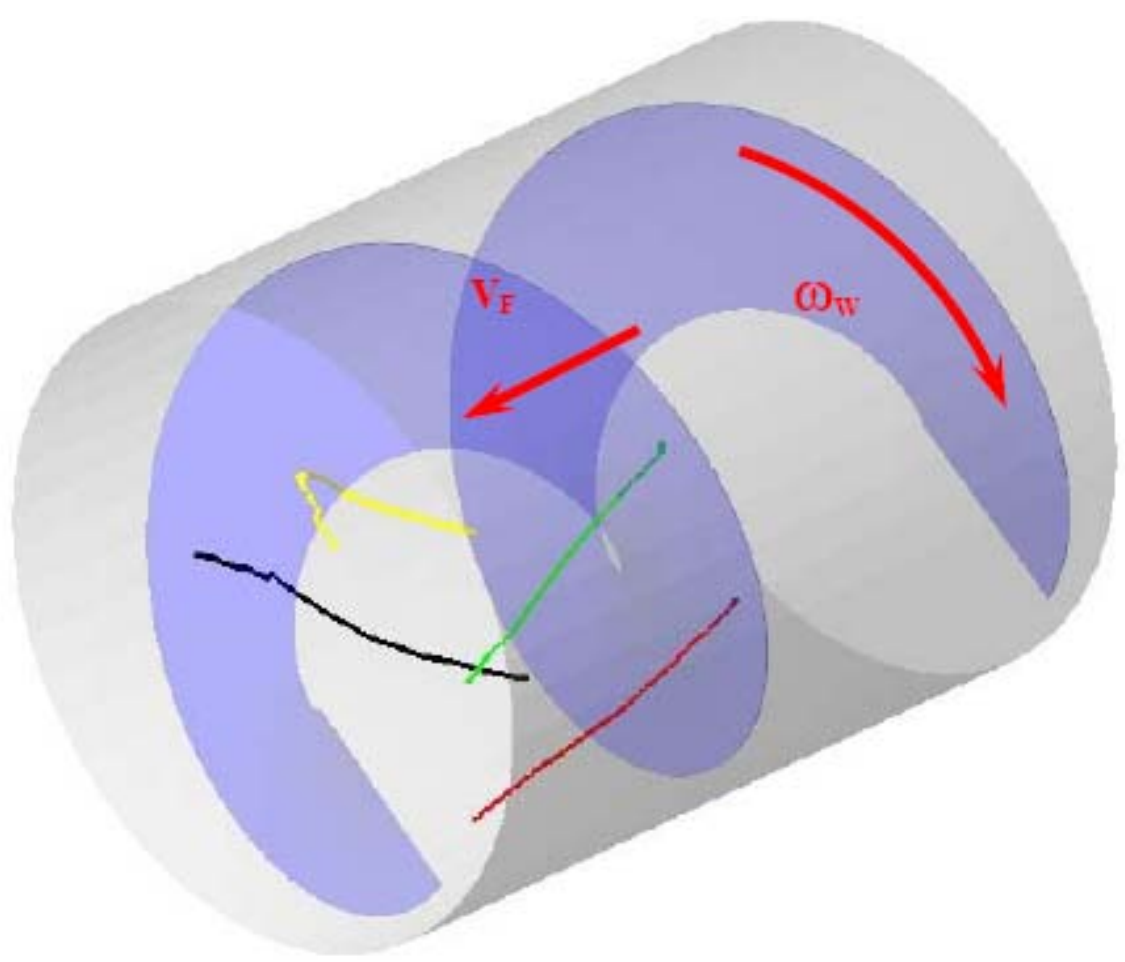

Abbildung 5: Pfade von 4 Partikel aus der DEM-Simulation des HWF des MVS bei $\eta_{F}=0,6$ und $n_{W}=46 \mathrm{~min}^{-1}$ 


\subsection{Ergebnisse der quantitativen Untersuchungen mit Hilfe der DEM für horizontale Wendelförderer mit Mischgranulat}

Die Abbildungen 6 und 7 zeigen den gesamten Verlauf des schüttgutabhängigen Drehmomentes $M_{F}$ im Lastlauf im Simulationsmodell des Wendelförderer-Modelversuchsstandes (Abb. 2) und des gemessenen Gesamtdrehmomentes $M_{W}=M_{W 0}+M_{F}$ im Wendelförderer-Großversuchsstand (Abb. 1) aus [Krause05a][Krause05e], [Minkin05]. Den gleichen Signalablauf-Charakter hat die Axialkraft $F_{a x}$ an der Wendel im modellierten und realen Wendelförderer.

Hier muß darauf hingewiesen werden, daß die Bilder quantitativ nicht vergleichbar sind, weil es um zwei Wendelförderer handelt, die unterschiedliche Geometrie- und Betriebsparameter haben. Das DEMSimulationsmodell wurde für den Wendelförderer des Wendelförderer-Modellversuchsstandes erstellt. Quantitative Untersuchungen am Wendelförderer-Modellversuchsstandes konnten bislang nicht durchgeführt werden, da er noch nicht mit entsprechender Meßtechnik ausgerüstet und darum nur für qualitative Untersuchungen genutzt wurde. Die Meßsignale der produktiven und mechanischen Kennwerte konnten bislang nur mit Hilfe des Wendelförderer-Großversuchsstandes aufgenommen werden (Abb. 7). Trotzdem können die Signale qualitativ verglichen werden. Aus den Bildern folgt, daß das Drehmoment im simulierten und realen Wendelförderer periodisch um einen Mittelwert schwankt. Das gleiche gilt auch für die Axialkraft $F_{a x}$ an der Wendel.

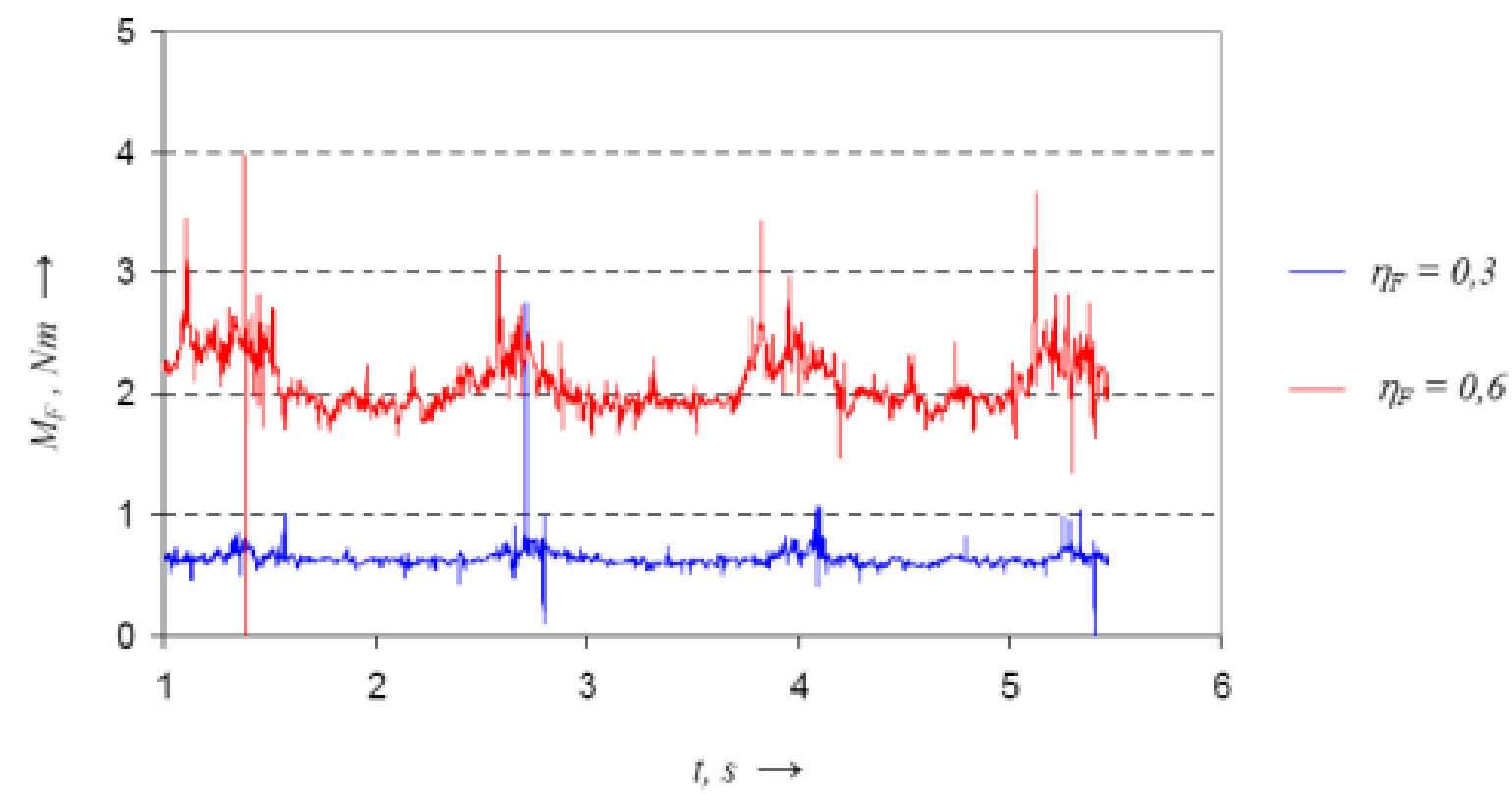

Abbildung 6: Verlauf des schüttgutabhängigen Drehmomentes $M_{F}$ an der Wendel in der DEM-Simulation des Wendelförderer-Modelversuchsstandes bei $\eta_{F}=0,3$ und $\eta_{F}=0,6$ und $n_{W}=46$ min $^{-1}$ mit verschiedenen Partikelgrößen. 


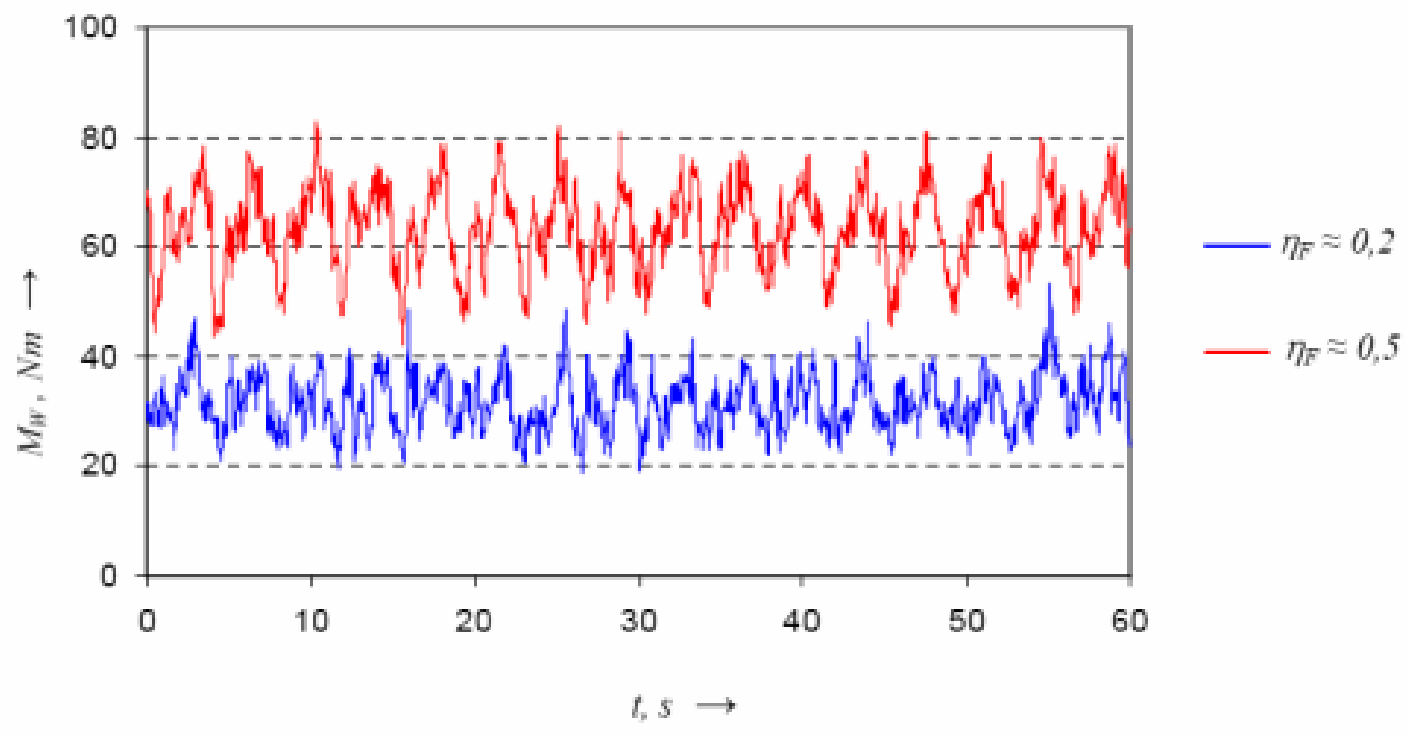

Abbildung 7: Verlauf des gemessenen Gesamtdrehmoments $M_{W}$ an der Wendel des WendelfördererGroßversuchsstandes bei $\eta_{F} \approx 0,2$ und $\eta_{F} \approx 0,5$ und $n_{W}=16 \mathrm{~min}^{-1}$ mit mit Mischgranulat.

Auf Basis der in [Krause05a]-[Krause05e], [Minkin05] formulierten Auslegungsrichtlinien konnten für HWF des Wendelförderer-Modellversuchsstandes das schüttgutabhängige Drehmomentes $M_{F}$ und die Axialkraft $F_{a x}$ an der Wendel für den Mischgranulat berechnet werden. Die errechneten Werte ermöglichen den Vergleich mit den aus der Simulation ermittelten Werten des Drehmomentes und der Axialkraft (Abb. 8).

Abbildung 8 zeigt, daß sich die in der Simulation ermittelten Werten des schüttgutabhängigen Drehmomentes $M_{F}$ und der Axialkraft $F_{a x}$ an der Wendel des MVS für Mischgranulat den anhand der Auslegungsrichtlinien vorhergesagten Werten annähren. An dieser Stelle muß darauf hingewiesen werden, daß die Berechnungsgrundlagen der mechanischen Kennwerte für HlgWF die schüttgutabhängigen empirischen Faktoren beinhalten, die anhand des HWF am GVS ermittelt wurden. Die Geometrieparameter eines Wendelförderers haben zwar nicht maßgeblichen aber trotzdem einen gewissen Einfluß auf die empirischen Faktoren. Der HWF des GVS weist einen U-Trog und eine Wendel auf, die aus zwei Wendeln zusammengeschweißt ist. Der modellierte HWF des MVS hat im Gegensatz dazu einen O-Trog und eine Einblattgeometrie-Wendel. Diese Geometrieunterschiede sowie noch nicht endgültig abgeschlossene Kalibrierung der Simulationsparamter erklären die Abweichungen der simulierten von den realen Werten.
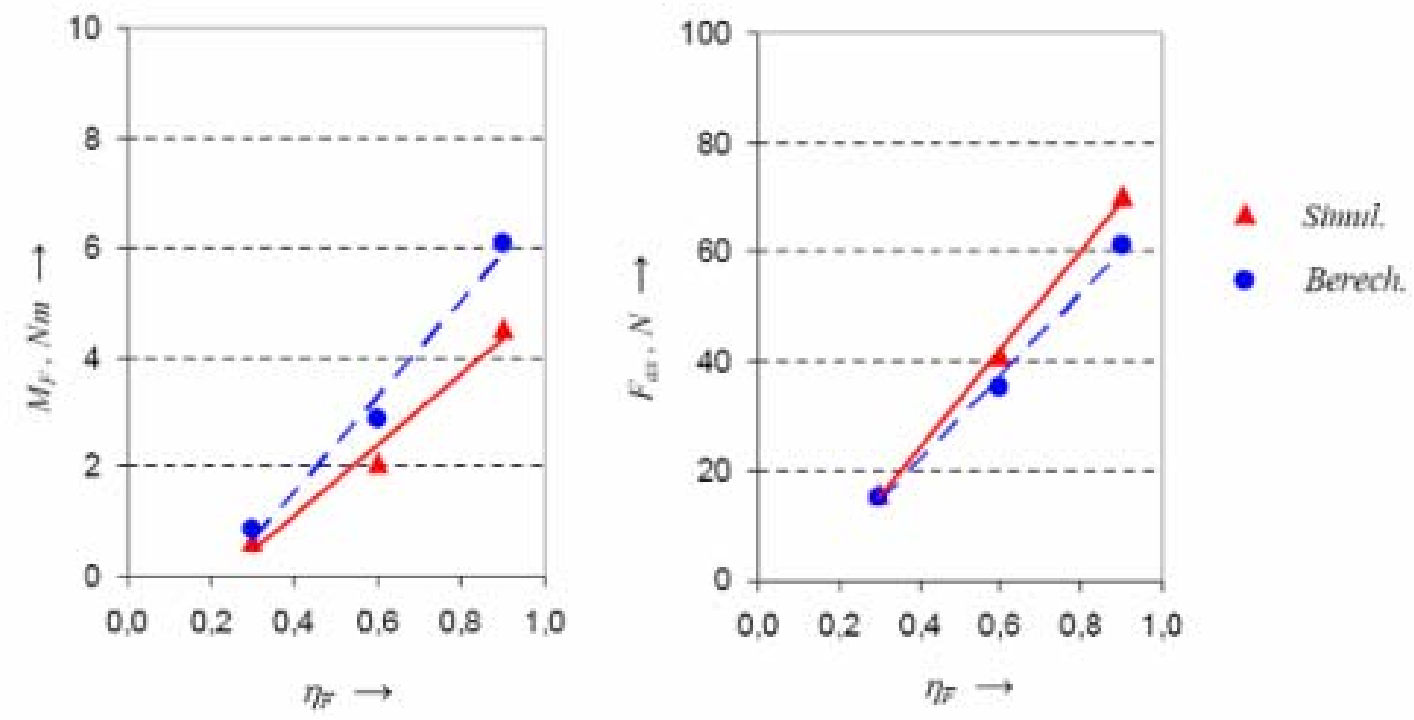

Abbildung 8: Simulierte und vorhergesagte Werte des schüttgutabhängigen Drehmomentes $M F$ und der Axialkraft $F_{a x}$ an der Wendel des Wendelförderer-Modellversuchsstandes für Mischgranulat in Abhängigkeit vom Füllungsgrad $\eta_{F}$. 


\section{Zusammenfassung und Ausblick}

Die in diesem Artikel präsentierten Ergebnisse der DEM-Simulation der Wendelförderung sprechen dafür, daß die DEM bei der Entwicklung und Planung von Materialflußtechnikanlagen sowie bei der Lösung technischer Probleme in der Materialflußtechnik ein leistungsfähiges Werkzeug ist.

Die Ergebnisse der qualitativen und quantitativen Untersuchungen eines horizontalen Wendelförderers mit Hilfe der DEM für Mischgranulat stimmen vorerst gut mit der Realität überein.

Allerdings wurde die DEM-Untersuchung im HWF für eines der „einfachsten“ Schüttgüter durchgeführt, da Mischgranulat ein leichtfließendes Schüttgut mit einem Körnern mit abgerundeten Kanten und ungefähr gleichen Abmaßen in den drei Dimensionen ist. In der Praxis bereiten solche Schüttgüter keine Probleme. Von der Industrie wird aber erwartet, daß DEM-Lösungen vor allem für die „schwierigen“ Schüttgüter zur Verfügung gestellt werden. Dazu gehören kohäsive und adhäsive, halbfeste und pastöse Güter. Dies kann nur durch aufwendige Kalibrierung der Simulationsparameter und Verifizierung des Förderverhaltens durch experimentelle Untersuchungen erfolgen, wodurch die Anpassung des Modells an die Realität gewährleistet wird. Für faserige, sich verstrickende Güter und Feststoff-Wasser-Gemische für die der Wendelförderer besonderen Einsatz findet, sind in absehbarer Zeit aufgrund der sehr schwierigen Modellierung dieser Güter und der Rechenintensität (gerade in Kopplung mit Computer-Fluid-Dynamic- (CFD-) Simulationen) keine direkten Simulationsergebnisse zu erwarten.

\section{Formelverzeichnis, Abkürzungen und Indizes}

\begin{tabular}{|l|l|l|}
\hline$F_{a x}$ & Axialkraft an der Wendel aus dem Schüttgut & $\mathrm{N}$ \\
\hline$I_{m}$ & Nennleistung & $\mathrm{t} / \mathrm{h}$ \\
\hline$M_{F}$ & Drehmoment infolge der Schüttgutreibung & $\mathrm{Nm}$ \\
\hline$M_{W 0}$ & Drehmoment im Leerlauf & $\mathrm{Nm}$ \\
\hline$M_{W}$ & gesamtes Drehmoment im Wendelförderer & $\mathrm{Nm}$ \\
\hline$T$ & Zeit & $\mathrm{s}$ \\
\hline$n_{W}$ & Wendeldrehzahl & $\mathrm{min}^{-1}$ \\
\hline$\varepsilon$ & Ablenkwinkel des Schüttgutkörpers aus der Ruhelage (Verdrehwinkel) & $\mathrm{grad}$ \\
\hline$\eta_{F}$ & Füllungsgrad & \\
\hline DEM & Diskrete Elemente Methode & \\
\hline GVF & Wendelförderer-Großversuchsstand Diskrete Elemente Methode & \\
\hline GWF & geneigter Wendelförderer & \\
\hline MVS & Wendelförderer-Modellversuchsstand & \\
\hline HWF & horizontaler Wendelförderer & \\
\hline HlgWF & horizontaler und leicht geneigter Wendelförderer & \\
\hline RKF & Rohrkettenförderer & \\
\hline VWF & vertikaler Wendelförderer & \\
\hline exp, th & experimentell bzw. theoretisch & \\
\hline
\end{tabular}

\section{Literatur}

[Gröger99]

[Gröger03]

[Günthner02]

[Katterfeld03]
Gröger, T.: Partikelmechanische Untersuchungen zur senkrechten Schlauchgurtförderung. Magdeburg, Otto-von-Guericke-Universität, Diss. 1999 Gröger, T.: Grundlagen der Diskreten Elemente Methode in der Schüttgutfördertechnik. Fachtagung Schüttgutfördertechnik 2003, Otto-vonGuericke-Universität Magdeburg.

Günthner, W.; Fottner, J.; Rong, O.: Empirische Entwicklung von Kenngrößen zur Auslegung von Hochleistungs-Schneckenförderern für Schüttgut. AiFForschungsbericht, München, FML, Technische Universität München, 2002.

Katterfeld, A.; Krause F.: Anwendung der Diskreten Elemente Methode in der Schüttgut-Fördertechnik, Tagungsband zur Schüttguttagung 2003, Verlag Logisch GmbH, Magdeburg 2003 
[Katterfeld04]

[Krause05a]

[Krause05b]

[Krause 05c]

[Krause05d]

[Krause05e]

[Krause05f]

[Minkin05]

[Rong04]

[Shimizu01]
Katterfeld, A.; Krause F.: Funktionsanalyse eines Rohrkettenförderers mit Hilfe der Diskreten Elemente Methode (DEM), Fachtagung Schüttgutfördertechnik 2004 Tagungsband, München, Technische Universität, 2004

Krause, F.; Minkin, A.: Ergebnisse der theoretischen und experimentellen Untersuchungen an horizontalen und leicht geneigten Wendelförderern. Teil 1: Theoretische Untersuchungen an horizontalen und leicht geneigten Wendelförderern. IN: Schüttgut.11(2005)3, S. 164-171

Krause, F.; Minkin, A.: Research on shaftless screw conveyors. IN: Bulk Solids Handling, (2005)2, S. 92-100

Krause, F.; Minkin, A.: Theoretical and experimental study of horizontal and lightly inclined shaftless screw conveyors. Part 1: Theoretical study of horizontal and lightly inclined shaftless screw conveyors. IN: Bulk Solids Handling, (2005)3, S. 172-178

Krause, F.; Minkin, A.: Theoretical and experimental study of horizontal and lightly inclined shaftless screw conveyors. Part 2: Quantitative study of horizontal and lightly inclined shaftless screw conveyors. IN: Bulk Solids Handling, (2005)4, S. 226-233

Krause, F.; Minkin, A.: Untersuchungen zur Wendelförderung. IN: Schüttgut, 11(2005)2, S. 99-111

Krause, F.; Katterfeld, A.; Minkin, A.: Zur Funktionsfähigkeit der Materialflußsystem-Elemente vor den Kollerpressen der MBV- /EBS-Anlage Stralsund. Unveröffentlichtes Gutachten, Magdeburg, Otto-von-Guericke Universität, 2005

Minkin, A.: Funktionsanalyse von Wendelförderern. Magdeburg, Otto-von-Guericke Universität, Diss. 2005

Rong, O.: Auslegungsverfahren für Hochleistungs-Schneckenförderern. München, Technische Universität, Diss. 2004

Shimizu, Y; Cundall, P.: Three-dimensional DEM simulations of bulk handling by screw conveyors. IN: Journal of Eng. Mechanics ASCE.127(2001)9, pp. 285-292 